\title{
Computational Approaches for Urban Design Within the MENA Region
}

\author{
Jaouad Akodad $^{\mathrm{a}}$, Mohammed BAKKALI ${ }^{\mathrm{b}}$ Mounir GHOGHO $^{\mathrm{c}}$ \\ a,b,c UIR Technology of Information and Communication Laboratory, Rabat, Morocco \\ ${ }^{a}$ National School of Architecture, Rabat, Morocco
}

\begin{abstract}
This paper focuses on the use of computational tools to develop a data driven approach for an analytical study about different urban systems. This "framework" examines urban Big Data in the old medina of Sale in Morocco. The computational tools are more effective to provide insights within complexity, becoming a key to generate more efficient solutions throughout the design process. The findings of this study highlight the potential of a data driven approach to explore analytical aspects and move further to generative design using algorithms.
\end{abstract}

Keywords. Parametric, Computation, Big Data, Urban Analytics

\section{Introduction}

The technology of information and communication (TIC) had an important impact in the development of technology as well as the design practice. Technology is always redefining new boundaries by the introduction of new tools that simulates architects and designers' creativity, allowing them to explore new concepts and innovative methods. The introduction of the TIC in the 60's for the design field had a significant impact by replacing the hand drawing tools with computational aided design (CAD). Afterwards, these tools had been improved as a base for architectural design, interior design, urban design and so on and so forth. They have a strong ability to produce efficient results and add a fresh perspective to these fields. The integration of this technologies on the design practice remains largely underexploited, presumably because technology is advancing faster than the construction industry. In order to benefit from the true potential of computation, this has to be implemented from the early stages of the design process, not only as a drawing tool, but introducing new possibilities in the use of computation in both the analytical phase and the design process through developing a very strong bond leading to a generative design where analytical data is exploited smartly. The possibilities derive largely from the increase of data control and creating new computeroriented approaches that benefit from the computer processing power. 


\section{Methodology}

This paper provides an analysis study of the historical medina of Sale in Morocco. Through computation, urban data will be implemented in a networked system (see, figure 1), generating insights about how the city's related systems respond and perform vis-àvis end-users' for today and tomorrow. The paper will start by setting the sources of data to be used (data mining), and how they are managed in a dynamic way using algorithms and visual programming (VP). The following chapter will focus on the construction of interlinked relationships between various form of data in order to produce meaningful visualisation of these systems. The goal is to provide a "framework" based on computer and human interactions and to seek to produce computational modelling so as to better examine urban big data in a frame of networked urbanism.

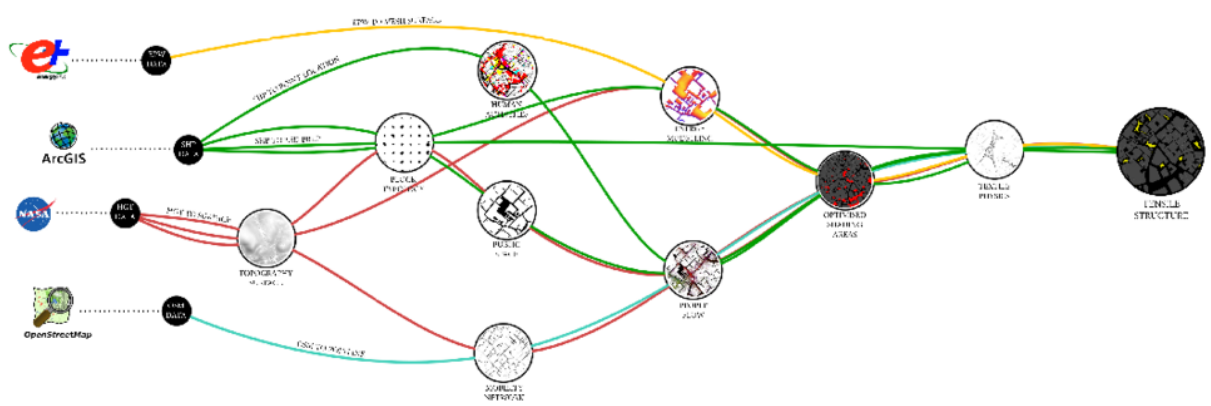

Figure 1. neural network methodology

\section{Data mining and the city}

Nowadays, cities become more complex and more dynamic to be apprehended making it unbearable for people to manually process data. At the same time, Big Data is increasing considerably, becoming more available and elaborated, and providing massive information about all types of behaviours and patterns within cities. Information extracted from Big Data and synthetized through Data Visualisation will enhance the level and accuracy of decision making and experts knowledge regarding different design choices. From the perspective of architects, urban designers and planners, and considering the tools with which they work in buildings and other design types, many aspects of data can be supported in the analytical phase such as geo-referenced data, which gives insight about spaces and their related information. It will support an evolved understanding of complex urban systems such as infrastructure networks, mobility, urban typo-morphologies, urban activities, energy modelling, topography and so on and so forth. 


\subsection{Image processing of historical maps}

In the past centuries, historical maps were largely produced manually, giving valuable information about land use at that time. Such cartographies can still provide useful data for historians, geographers, and planners to comprehend different historical aspects.

Much research has been made concerning historical maps. Jessop and Rumsey and Williams [4]. investigated the use of Geographic Information Systems (GIS) to analyse historical maps and layering them with other spatial data. Balletti [5] proposes some efficient methodologies for quantitative analysis of the metrical content of historical maps. This chapter proposes an investigation of the historical maps in the specific site of the medina of Sale, mainly produced under the French protectorate. This work aims to study the urban evolution of the spot through Image processing. In the first stage, a data mining is proceeded. The goal is to collect a considerable number of cartographies, which will be scanned and stored in digital files. In the context of the Medina of Sale, the cartographies highlighted few concerns on maps from the $18^{\text {th }}$ century until 2018 , a period when the historical site went through major transformations. The cartographies are rescaled afterwards and overlapped in a matrix of pixels. Cartography corresponds to a large number of symbols, colours and observations displayed on a constrained screen with limited resolution. Based on this view, building symbols can be selected through their pixel's values. The next step consists in writing an algorithm that maps each square in the matrix with the number of overlapping layers. For example, a square was (clarify here please) 1 layer of building is overlapped, will correspond on value of 1 , with 2 layers the value will be 2 and so on and so forth. Finally, a colour gradient is applied to visualise properly the results depending on the values of each pixel. The use of image processing in a complex site proved an effective result on representing urban evolution. The innovative use of image processing allows the computation of high number of maps and visualise meaningful information, and providing experts with useful knowledge on the city's growth. The results may be improved using additional cartographies. Some obstacles had to be overcome while extracting information from images of historical maps. The layering of image maps had to be slightly adjusted because of the accuracy of the hand-made cartographies provided. Also, the level of accuracy was highly related to the image resolution.
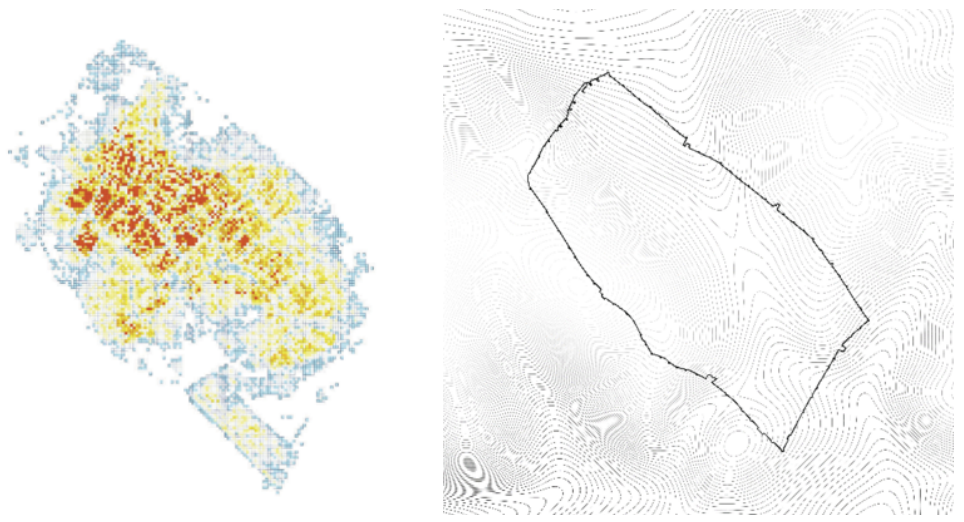

Figure 2. Mapping Urban Evolution (left) and Topography visualization (right) 


\subsection{Topography}

Shuttle Radar Topography Mission (SRTM) data are used to reconstruct the topography of large regions, which consist of Digital Elevation Models generated by NASA and the National Geospatial-Intelligence Agency. SRTM topographic data consists in 90-meter (295-foot) pixels spacing for elevation's information of many part of the world. Figure 2 illustrates the generation of topographic levels of Sale city that correspond to (N34 W006). Within the visual programming platform of Rhinoceros, many scripts are provided to help convert Hgt (explain Hgt) files into geometrical surface, and then, applying horizontal contours in order the visual the topography curves.

\subsection{Morphology}

Two dimensional (2D) urban morphology was provided from CAD files and managed into a Shapefile (explain a shapefile in what it consists of) to contain the elevation data. The idea is to extrude each plot with the corresponding elevation value in order to construct a $2.5 \mathrm{D}$ model (see, figure 3 ).

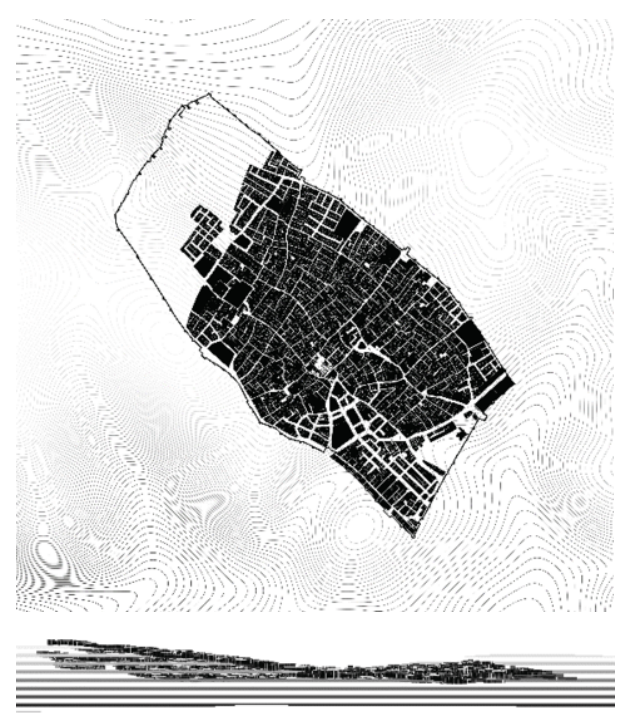

Figure 3. Morphology visualization 


\subsection{Infrastructure Network}

Open Street Map in a non-profit organization providing geographic diverse user-based data of addresses, places' names, and route planning. Once the data has been collected, it is entered into an algorithm that manages the infrastructure network. Figure 4 visualizes how mobility is distributed from highways to pedestrian pathways.
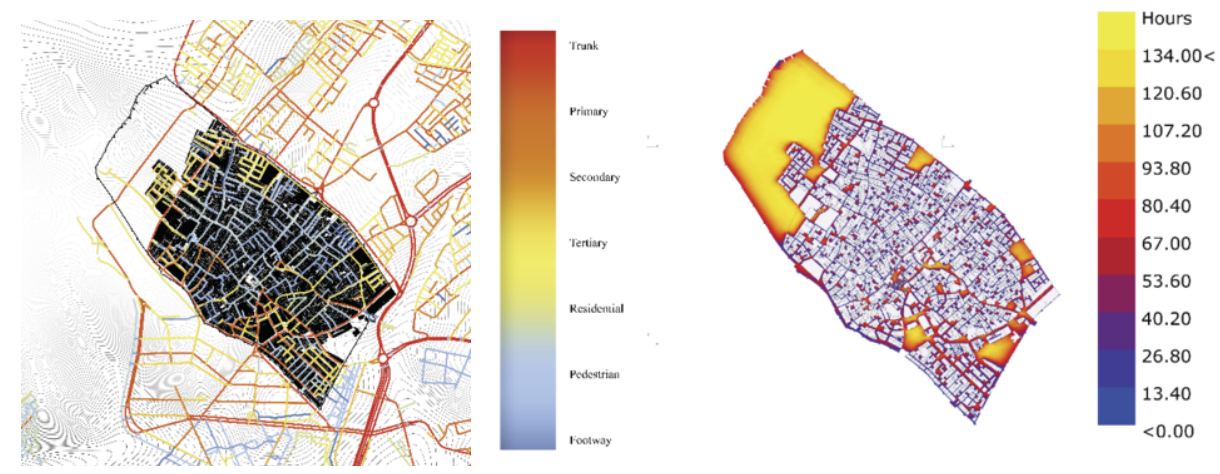

Figure 4. Mobility Network visualization (left) and Sun Hours analysis (All days of December - right)

\subsection{Energy analysis:}

Recently, many tools allowed new approaches of energy modelling and thermal simulation. Ladybug is an open-source plugin implemented in Rhinoceros/Grasshopper to help designers explore relationships between environmental data and buildings geometry models. The process of making energy models within the design platform is more effective to support complex geometries, i.e. the urban morphology of a historical medina. The using of energy modelling to endorse environmental analysis within architectural practices has been noticed to reveal more useful solutions when coupled with design processes. The generation of graphical data such as the sun path, sunlight hours, radiation studies, and more are proceeded by providing specific inputs. In one hand, Energy Plus with weather data (EPW ) of the city of Sale. In the other hand, the geometry of the city, which is the $2.5 \mathrm{D}$ model of the medina. In order to achieve more effective results, a projection will be applied to the geometry geo-located at the surface of the topography levels (see, figure 4). 


\subsection{Local Activities}

Applied to the context of the medina of Sale (see, figure 5), we provide various types of local activities, integrated in a database-oriented Geographic Information System (GIS). To increase control of this massive data, a classification is made to construct five major themes: Artisanal, retail, cultural, and touristic activities.

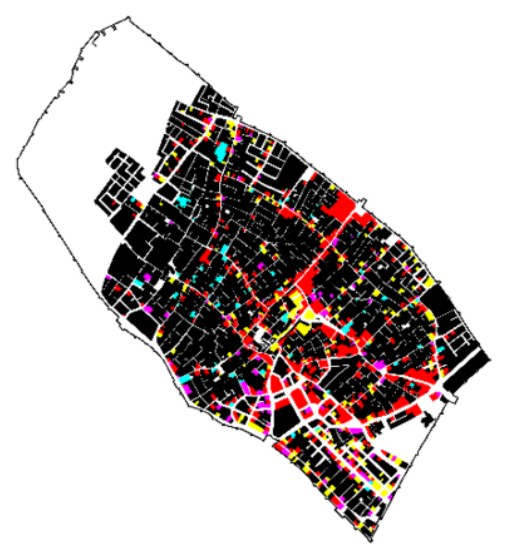

Figure 5. Local activities visualization

\section{Generative process through a networked system}

This chapter focus on identifying interlinked relationships between the various sources of data to explore how the city is performing, by integrating them into a networked system based on algorithms. According to this view, the city is seen as a set of complex interactions between its different urban components, which is mainly incorporated in the concept of the Smart City. The urban Big Data become a tool to better inform planners about how the city is responding by giving insights from very large and dynamic datasets. The proposed method proposes consecutive phases, gathering data coming from different sources, and implemented into an algorithm. They are summarized in three main phases:

- Definition of thematic activities.

- Investigation of public spaces.

- Determination of people flow.

Decomposing and analysing parts of the medina is significant to reveal patterns of its urban systems. In this process, the traditional techniques may not be capable to evaluate such complex activities neither to deal with different parts acting simultaneously. Meanwhile, using computational tools can process such complexity, and manage human activities into thematic classification to form six main themes: Retail, Artisanal, Tourism, and Culture. The thematic classification can serve to investigate the behaviour of public spaces that mainly serve to the growth of informal activities. The term "Informal activity" is this study is not considered as a spatial activity taking place randomly around 
the public space, but more related to a harmonious growth, primarily fed by the existing urban systems. Thus, the evolution of informal activities through time is related to city patterns, that are specific to a domain from where data is collected. By integrating the distribution of the thematic activities into the algorithm, a zone of people moving in and out can be predicted by connecting all the locations of each thematic classification. The people flow visualization is based also on the existing urban roadways that provide information about several hierarchical levels of the mobility network. Furthermore, the people flow will serve to determine the zone where informal activities self-evolve, while public places are considered as the centre of growth of each thematic activity. Whenever a block level is null and its size is larger than $200 \mathrm{~m}^{2}$, the urban typology is referenced as public place. The investigation can also examine the potential of each public space to become the centre of a thematic activity. Through computational processing, the visual analytic explores how the city is performing by setting relationship between the public places, the people flow, and the location of classified activities. The next stage on the design process consists of the generative process based on the network system output and additional parameters, such as the energy modelling that will serve mainly to evaluate the thermal simulation on the path of people flow. The application of energy simulation in this process aim to study the solar shading in public spaces, in order to optimize long term outdoor thermal comfort. Therefore, the existing building and topography must be considered when creating shading outdoor areas. In addition, the EnergyPlus provides Data (EPW format) from exact locations to study the solar path of all periods of a day, month, or the whole year. The analytical study results on areas that necessities sufficient shading while avoiding excessive shading that are already created from the surrounding environment. Finally, the resulting areas that need to be shaded, are converted to mesh surfaces, and implemented to a plugin (Kangaroo) within grasshopper platform, to apply physics of textile. The surrounding buildings will serve as anchors, by connecting the mesh surface to the closest building corners. Additional parameters are also taken into account such as gravity force, and the spring strength. Tensile structures are the result of the generation process, which are applied to each thematic path, generating a multitude of shapes mainly related to environmental parameters. visual analytics served to specify areas that necessities a design intervention, rather than applying the design to the whole site (see, figures $6-8$ ).

\section{Results}

This study represents the findings derived from the use of computational approach applied to the urban design framework. The method gathers visual analytics of urban Big Data which furthermore serves as inputs to a generative design. The historical context of the Sale medina demonstrates the relevance of this approach to reveal the complexity found within its urban systems. The early stage of the design consists in data visualisation of urban pattern such as the topography, mobility network, urban typologies, and human activities. It is essential to take into consideration those urban patterns in every stage of the urban design, and to inform the design generation using a data and computation. What is more is the capacity to increase control of the data collected, while integrating them into a networked system, within the Rhinoceros/Grasshopper platform. As demonstrated in this study, the environmental data serves as input to develop, generate, optimized membrane structures in the public space of the medina, based on a data driven process. The key to a more efficient designs combines the computer processing constructed to 
manage connections between the information collected from the analytical study and inform the design formation. The visual analytics served to specify areas that necessities a design intervention, rather than applying the design to the whole site.

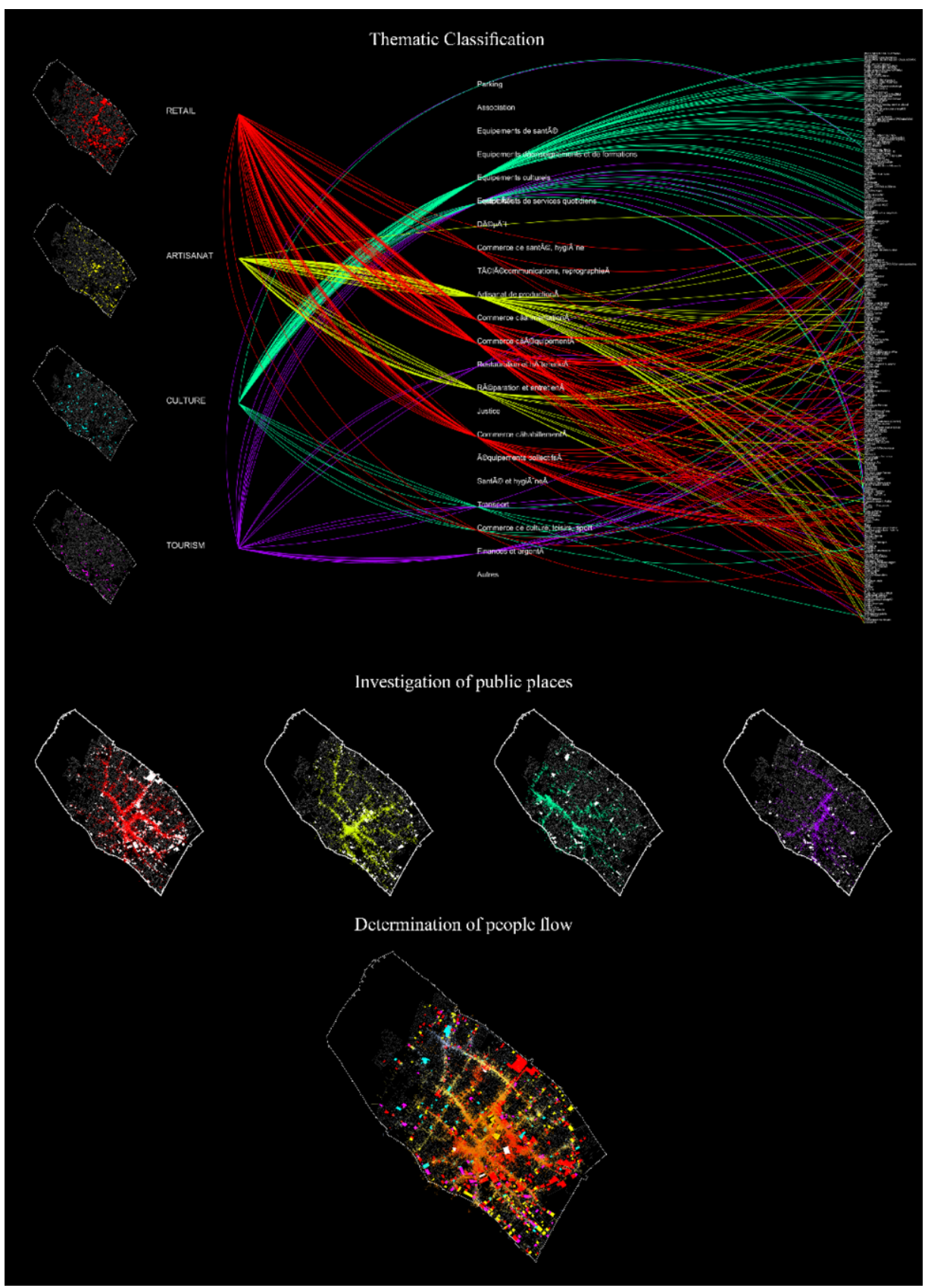

Figure 6. Visual Analytics 


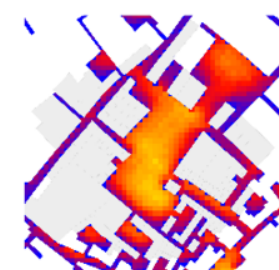

Thermal simulation
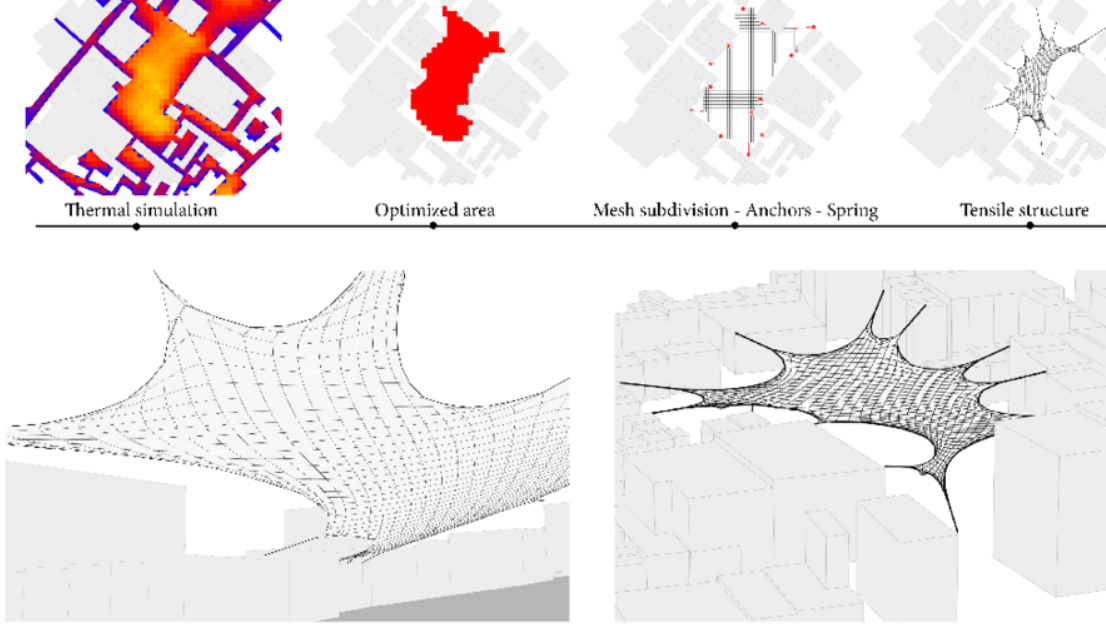

Mesh subdivision - Anchors - Spring

Tensile structure

Figure 7. Generative design of tensile structure

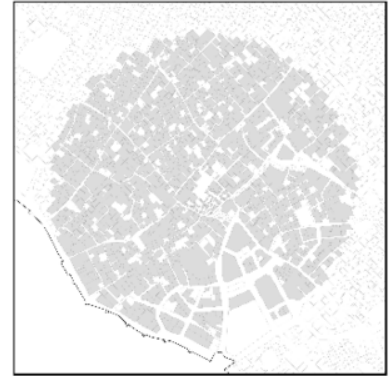

1- Arca of study.

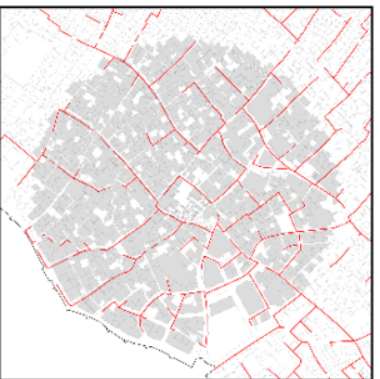

4- Path of pcople flow

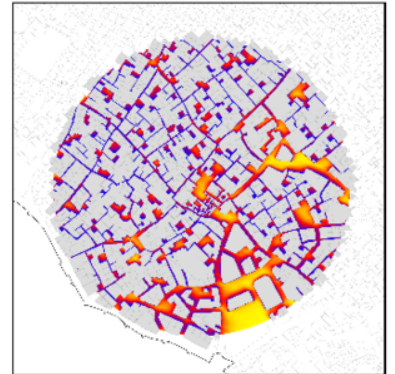

2-Shading Simulation (8am-19pm/12month)

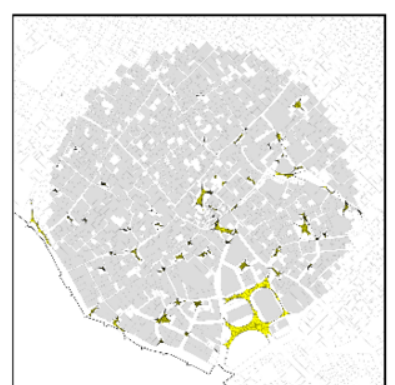

5- Tensile structure generation

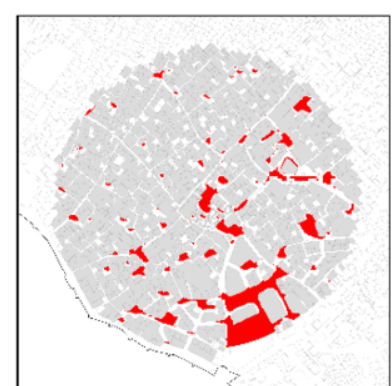

3- Zone to gencrate shading

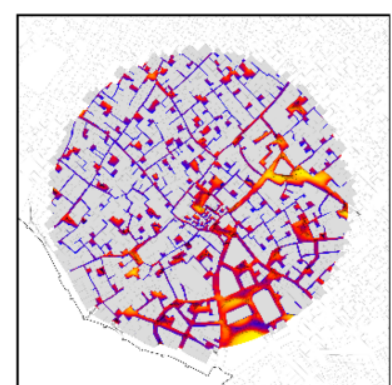

6- shading simulation after generation

Figure 8. Step by step of the design generation process 


\section{Conclusion and future work}

The framework based on algorithms and visual programming shows its ability to better control large amount of data city from various sources, within a unified interface. It is become essential to consider a data driven approach in the design methodology, especially with the increase of data production that are giving added value in term of informative insights and knowledge to guide decision making and inspire for further limitless development that can be found in its application. Urban Big Data should be increasingly available in the forthcoming years. The analysis phase could integrate image base data (like satellite images). It could be a powerful tool to manage the city patterns use of image recognition, and better inform urban planning and designers. Computing processing could also be extended in the generative process of urban planning and design. For instance, the use of shape grammar shows a potential to work with geometry and rules. These rules could be linked to urban parameters and informed from city data, providing the ability to generate shapes according to this specific area.

\section{Acknowledgment}

This study would not be possible without the support from the UIR Technology of Information and Communication Laboratory under the PV Build Project. I would like to thank my supervisors Prof. Mohammed BAKKALI and Dr. Mounir GHOGHO for their guidance and support throughout the study.

\section{References}

[1] Ackoff, R. L. From data to wisdom. Journal of Applied Systems Analysis. 1989: 16, 3-9.

[2] Carlo Ratti, Paul Richens. Urban Texture Analysis with Image Processing Techniques. The Martin Centre for Architectural and Urban Studies, University of Cambridge. UK; 1999;

[3] Christina Martelli, Emanuelle Bellini. Using Value Network Analysis to support Data Driven Decision making in Urban Planning. Conference: Proceedings of the 2013 International Conference on Signal Image Technology and Internet-Based Systems. 2013 Dec:112-6

[4] Jessop, M (2006). Promoting cartographic heritage via digital resources on the Web. ePerimetron vol.1/3: p. $246-252$.

[5] Balletti, C. Georeference in the analysis of the geometric content of early maps. ePerimetron vol.1/1: p. $32-42$. (2006).

[6] Mostapha Sadeghipour Roudsari. Ladybug: A parametric environmental plugin for grasshopper to help designers create environmentally conscious design. 2013 Jan: 3128-3135.

[7] Pınar ÇALIŞIR ADEM, Gülen ÇAĞDAŞ. Interpretation of urban data in the complex pattern of traditional city: The case of Amasya. Vol 15 No 1, March 2018: 23-38.

[8] Rob Kitchin. Data Driven, Networked Urbanism. SSRN Electronic journal. 2015. 